Fecha de recepción: julio 2011 Fecha de aceptación: octubre 2011 Versión final: abril 2012

\section{Comunicación y Cultura: análisis de la realidad de las Relaciones Públicas en organizaciones chilenas y brasileñas ${ }^{1}$ \\ María Aparecida Ferrari *}

\begin{abstract}
Resumen: El presente texto examina la práctica de las relaciones públicas y el rol que desempeñan los profesionales que administran el área en organizaciones chilenas y brasileñas, según los resultados de la investigación Influencia de los valores organizacionales en la práctica y el papel de los profesionales de las relaciones públicas: Estudio Comparativo entre organizaciones de Brasil y Chile realizada por Ferrari (2000). Aborda conceptos de comunicación y relaciones públicas que llevan a entender que la legitimidad de las dos áreas está asociada a factores políticos, económicos, sociales y culturales locales. Los resultados arrojados por el estudio apuntan que gran parte de las organizaciones, sea en Chile o en Brasil, se encuentra en transición de una cultura autoritaria para una cultura participativa, una evolución asociada a factores como el fenómeno de la globalización de los mercados, los nuevos modelos de gestión, la apertura económica, la estabilidad política y una mayor participación de los ciudadanos en la vida empresarial. A pesar del desarrollo que las organizaciones en Chile y Brasil han alcanzado en la última década, los resultados arrojados siguen válidos, según demuestra estudio en marcha por Ferrari (2010).
\end{abstract}

Palabras clave: comunicación - cultura - cultura nacional - cultura organizacional - organización - Relaciones Públicas - valores organizacionales.

[Resúmenes en inglés y portugués en las páginas 63-64]

${ }^{(*)}$ Doctora en Ciencias de la Comunicación. Docente en programas de postgrado y pregrado en el área de la comunicación en diversas universidades latinoamericanas. [Ver CV completo en la página 140]

\title{
Introducción
}

Hasta el inicio del siglo XXI, las Relaciones Públicas aún no se practicaban como una función estratégica en las estructuras de las organizaciones en América Latina. La actividad carecía del reconocimiento de la sociedad que las relaciones públicas son indispensables para el crecimiento de los negocios y para el establecimiento de relacionamientos más duraderos con sus públicos. El proceso de consolidación de las relaciones públicas en la región ha sido largo y tardío, lo que hace pensar que su desarrollo y legitimidad están asociados a factores políticos, económicos, sociales y culturales locales. Cabe señalar que en gran parte del continente latino- 
americano -donde un $80 \%$ de las empresas son de mediano y pequeño tamaño y familiares-las grandes organizaciones (sólo un 20\% del total de empresas) son multinacionales globales en cuya estructura se inserta la actividad de relaciones públicas. Aunque las multinacionales gocen de gran visibilidad por su dimensión e importancia, hay que reconocer que son las medianas y pequeñas empresas las que absorben un $80 \%$ de la fuerza laboral. Por esta razón, destacamos que la práctica de la comunicación en las multinacionales no es la misma ejercida en las medianas y pequeñas empresas nacionales.

Al contrario del contexto norteamericano, en que desde su inicio la práctica de las relaciones públicas fue incorporada en las estructuras organizacionales de la mayoría de las empresas y órganos gubernamentales, en América Latina la actividad se insertó de manera muy distinta en el universo corporativo. Tradicionalmente, en los países de la región, la práctica se introduce en las empresas multinacionales que se establecen en el continente sudamericano a partir de 1930, y que traen en sus organigramas el departamento de relaciones públicas - una función hasta entonces completamente desconocida entre los profesionales locales. Un análisis de ese proceso nos permite identificar diferencias estructurales y teóricas que marcaron la trayectoria de las relaciones públicas en la región. Desde el principio se hizo evidente el desequilibrio entre la práctica de las relaciones públicas en Norte América y Europa y en los países de América Latina. Las prácticas de relaciones públicas introducidas por esas recién llegadas empresas multinacionales, además, carecían de adaptación local, por lo que mucho de lo que se practicaba era falto de sentido en el nuevo contexto latinoamericano.

A pesar de algunos reconocidos avances, la difusión de la actividad quizás se haya frenado por el escaso intercambio entre los académicos y profesionales locales y por la falta de integración del continente, que se podría explicar por diferentes razones: la barrera cultural que resulta de la adopción de dos idiomas diferentes -el portugués en Brasil y el español en todos los demás países de América del Sur- que dificulta la asimilación de conocimientos entre investigadores de uno y otro idioma; y el rechazo de la propia identidad al importarse modelos foráneos como si ésos fueran ideales para la sociedad latinoamericana, ignorándose las prácticas culturales locales. Más recientemente, otros obstáculos han contribuido para debilitar la visión local positiva de las relaciones públicas: los problemas económicos y políticos de la región, el acceso disparejo de los ciudadanos a la tecnología; los exiguos recursos financieros y humanos para inversión en investigación en los centros académicos; la baja calidad de los programas ofrecidos por las universidades - mayormente en el área de comunicación, y la reducida actividad de investigación y de publicaciones locales.

Así, las relaciones públicas como práctica profesional, entendidas como fruto de los contextos político, económico, social y cultural de cada país, se han desarrollado de manera singular en cada uno de esos dos países, aunque algunos académicos (Pérez Senac y Solórzano, 1999) sugieran la existencia de una corriente latinoamericana de relaciones públicas.

\section{Conceptos de Comunicación y Relaciones Públicas}

Para iniciar el debate acerca del comportamiento comunicacional de las organizaciones chilenas y brasileñas conviene hacer una reflexión acerca de dos conceptos subyacentes: Comunicación y Relaciones Públicas. Tanto en la literatura especializada como en proyectos de investiga- 
ción empíricos hemos observado que esos conceptos tienen connotación y aplicación distintas en los países de América Latina. Quizás porque el desarrollo de esas áreas en el contexto latinoamericano no haya sido equilibrado y haya tomado rumbos diferentes, como es el caso de Brasil. Ferrari (2004) comenta que

uno de los hitos de más relevancia en la historia de las relaciones públicas en Brasil fue la creación de la ley 5.377 el 11 de diciembre de 1967, reglamentada por el decreto no. 63.283 del 26 de septiembre de 1968, que instituyó la profesión de Relaciones Públicas, hecho que transformó a Brasil en el primer país del mundo en adoptar una legislación específica de Relaciones Públicas que definía el concepto legal de Relaciones Públicas, sus funciones, la profesión, y fijaba las condiciones para el registro profesional y la fiscalización de la profesión (Ferrari, 2004, p. 57)

En cambio, no hubo la misma preocupación en legislar sobre la disciplina en los demás países de la región, salvo recientemente, en Panamá (1990) y en Perú (2005), que emularon la trayectoria de Brasil.

Mientras algunos teóricos adoptan el concepto de comunicación para explicar procesos como burocracia y persuasión, otros autores contemporáneos lo asocian a instrumentos mediáticos y espacios de los medios de comunicación.

Según Grunig, J., Ferrari, M. A. y França, F. (2009),

la comunicación es un proceso continuo y permanente que existe independientemente de la voluntad del hombre. En un primer momento ocurre de manera involuntaria; en seguida, a los comunicadores les corresponde entender el significado de la comunicación en la organización para, a continuación, planificarla para que su proceso sea adecuado a las necesidades de los públicos (Grunig, Ferrari, França, 2009, p. 157)

El proyecto de investigación llevado a cabo en organizaciones brasileñas y chilenas reveló que el término comunicación crea confusión entre los ejecutivos; para la mayoría de ellos, comunicación es sencillamente una herramienta para lograr objetivos establecidos en el plan estratégico; además, muchos confunden la comunicación con relaciones públicas, y desde esta perspectiva los dos términos son sinónimos. Los ejecutivos, pues, dan poca o ninguna atención al enfoque amplio de la comunicación como campo de conocimiento, más allá de su utilidad como herramienta operacional. La alta dirección no entiende la organización como fruto de la interacción de personas promovida a través del proceso de comunicación, al que las personas aportan sus expectativas y anhelos sobre su quehacer profesional y personal por medio de los relacionamientos. Nuestra visión de la comunicación como forma de comportamiento social la ubica en el centro de todo tipo de relacionamiento, sea en el interior o fuera de la organización.

Asimismo, admitimos que existen problemas conceptuales con la definición de comunicación, quizás por su complejidad, la definición de relaciones públicas enfrenta barreras más profundas de aceptación entre académicos y profesionales.

Cuando analizamos el constructo relaciones públicas, nos damos cuenta de que la gran cantidad 
de definiciones ha servido más para obstaculizar que para promover la disciplina, principalmente en términos de su función, lo que puede haber perjudicado el avance de las relaciones públicas como una especialidad de departamentos en las organizaciones.

Una alternativa de análisis de las relaciones públicas es focalizar el concepto como un fenómeno único en cada uno de los diferentes espacios sociales - continentes, regiones, países, culturas; así, la búsqueda de un denominador común para conceptualizarla es algo inútil (Van Ruller y Vercic, 2003).

Otra vía para intentar entender la disciplina es describir y explicar una organización desde la perspectiva de las relaciones públicas, como lo hace el marketing (a través de la perspectiva del mercado), el sector jurídico (desde el enfoque legal), o el sector financiero (desde la perspectiva de la relación beneficio/pérdida). Abordadas de esa manera, las relaciones públicas deben desarrollar un camino propio o una forma específica de pensar las organizaciones y, para eso, es necesario desarrollar una teoría desde la perspectiva de los relacionamientos.

Si relaciones públicas son más que relacionamiento, pues también atañen a la administración, el marketing y otras disciplinas, entonces, según Van Ruller y Vercic, "las relaciones públicas tienen un interés especial por los grandes asuntos de la sociedad y tratan cualquier problema desde una perspectiva de las implicaciones del comportamiento organizacional sobre la esfera pública y viceversa" (Van Ruller y Vercic, 2003, p. 167).

En la práctica del mercado, las relaciones públicas deben entenderse como una actividad de gestión de la comunicación dedicadas a: a) analizar los escenarios interno y externo de las organizaciones; b) tener como foco las relaciones con sus públicos estratégicos y, c) determinar la actuación de la organización hacia esos públicos sin desestimar el equilibrio de los intereses de ambos lados. Esas funciones están directamente relacionadas con la nueva visión de organización y de comunicación.

En su sentido más amplio, relaciones públicas es la actividad responsable por la construcción y mantenimiento de redes de relaciones entre las organizaciones y sus diferentes públicos. Desafortunadamente, en la Región bajo estudio, las relaciones públicas se han practicado desde una perspectiva mucho más operacional y táctica que estratégica; eso es lo que pretendemos demostrar con el presente proyecto de investigación llevado a cabo en ambos países.

\section{Estudio sobre la realidad de las Relaciones Públicas en organizaciones chilenas y brasileñas}

El resultado del estudio intercultural desarrollado por Ferrari (2000) buscó identificar y analizar la actividad de relaciones públicas y el papel desempeñado por los profesionales en 35 organizaciones, 22 brasileñas y 13 chilenas. La investigación cubrió 35 CEOs y 35 ejecutivos de comunicación. La muestra fue extraída de los rankings de la revista chilena Gestión y de su equivalente brasileña, Exame. Fueron seleccionadas, de manera aleatoria, organizaciones con departamento de Relaciones Públicas o Comunicación, teniendo en cuenta la diversidad de la nomenclatura de los sectores en los países estudiados. La muestra estuvo compuesta por organizaciones de diferentes sectores económicos como: químico, farmacéutico, alimentos, financiero, productos de aseo personal, minería y metalurgia, tabaco, petróleo y electrónica. Para cumplir el objetivo propuesto, se utilizó un cuerpo teórico conceptual basado en teorías y 
enfoques como la tipología de los cuatro modelos de práctica de relaciones públicas propuesta por Grunig y Hunt (1984), la tipología de los papeles desempeñados por los profesionales de relaciones públicas introducida por Broom y Dozier (1986) y por Wright (1995), características de la cultura nacional y cultura organizacional en los dos países estudiados, y la escala de valores organizacionales validada por Tamayo (1994) y complementada por Rodríguez (1991, 1995, 2001).

\section{La tipología de práctica de Relaciones Públicas por Grunig y Hunt (1984)}

En 1984, Grunig y Hunt propusieron cuatro modelos de práctica de relaciones públicas tras investigar organizaciones norteamericanas durante casi una década. Los modelos formulados por Grunig y Hunt (1984) corresponden a cuatro formas típicas de entender la naturaleza y el propósito de las relaciones públicas. Para los autores, los modelos constituyen descripciones simplificadas de la realidad y, por esa razón, tienen limitaciones. En los dos primeros modelos la práctica de las relaciones públicas consiste en una función técnica o táctica; en los dos últimos modelos la práctica tiene carácter gerencial y estratégico. Algunos de esos modelos son más eficaces o más éticos que otros, según la modalidad de gestión adoptada, del sector de actividad económica y del grado de vulnerabilidad del entorno específico.

En el primer modelo, agencia de prensa/difusión, la información fluye en sentido único: de la organización a sus públicos. Se trata de la forma más sencilla de practicar las relaciones públicas, y el modelo se acerca a la práctica de la publicidad porque el énfasis está puesto en la promoción de los servicios y/o productos. Este modelo de práctica está muy diseminado en los medios publicitarios, en los deportes, entre actores de cine y televisión, políticos, entre otros.

La información pública es el segundo modelo de práctica y difiere del primer modelo porque, en vez de presionar para vender, busca informar, aunque la comunicación para la diseminación de la información siga siendo de flujo único: del emisor hacia el receptor. Típicamente, la organización contrata a un periodista in house, o a relacionistas que actúan de periodistas para diseminar informaciones relativamente objetivas utilizando los medios de comunicación masiva, Internet o medios dirigidos, como newsletters, folletería y otros instrumentos. Según los autores, el modelo suele aplicarse en órganos gubernamentales y escuelas, ONGs y otras organizaciones. Tanto el modelo de agencia de prensa como el segundo modelo de información pública presentan un solo flujo, en que se elaboran y se ponen en práctica programas de comunicación sin que se haya conducido una investigación o un análisis del escenario.

El tercer modelo, asimétrico de doble flujo, también conocido como modelo de persuasión científica, utiliza la investigación para identificar las percepciones de los públicos para luego introducir la persuasión en los esfuerzos de comunicación. Los profesionales de relaciones públicas utilizan entrevistas, encuestas y grupos focales para diagnosticar las actitudes de los públicos que permitirán a la organización diseñar programas de relaciones públicas que le aseguren beneficios.

Este tipo de retroalimentación es parte del proceso, y la organización está netamente interesada en que los públicos ajusten sus expectaciones a las de la organización, y no al revés. Este modelo asimétrico es más eficaz que los dos anteriores para identificar la opinión de los públicos, precisamente porque introduce el elemento de retroalimentación, aunque ésta busque privilegiar 
los intereses de la organización.

El cuarto modelo, simétrico de doble flujo, está centrado en el entendimiento mutuo y en la comunicación de doble sentido en lugar de la persuasión, y recurre a la comunicación para administrar conflictos y mejorar la comprensión con los públicos estratégicos.

El modelo, amparado en la simetría, propone tres estrategias: la negociación, el consenso y la mediación, y está considerado como la práctica más ética entre todos los modelos presentados anteriormente.

Los profesionales de relaciones públicas que practican ese modelo actúan como mediadores entre la organización y los públicos de su entorno.

Desde luego, el modelo simétrico de doble flujo constituye un eje vertebral de la teoría contemporánea de las relaciones públicas, puesto que es considerado la práctica ideal de las relaciones públicas. A pesar de que Grunig (1992) lo define como el modelo normativo por excelencia, precisamente por su ubicación teórica, el modelo simétrico de doble flujo ha sido blanco de un sinnúmero de críticas, revisiones y adaptaciones desde que fue formulado por primera vez.

\section{La tipología de los papeles desempeñados por los profesionales de relaciones públicas desarrolladas por Broom y Dozier (1986) y por Wright (1995)}

Los roles desempeñados por los profesionales de relaciones públicas han sido objeto de investigación llevada a cabo por Broom y Smith (1979), Broom y Dozier (1995) Dozier (1992), Creedon (1991) y Dozier y Lauzen (2000).

Según Dozier (1992, p. 327), los roles "son abstracciones de padrones de comportamiento de los individuos en la organización". El autor añade que el papel desempeñado por el profesional está vinculado al modelo de relaciones públicas que se decida practicar. Así, y con base en la teoría de los cuatro modelos de Grunig y Hunt (1984), los profesionales quienes adoptan los modelos de agencia de prensa, divulgación e información pública, desarrollan habilidades dirigidas a técnicas que no exigen conocimiento de gestión o de estrategia. En cambio, los profesionales quienes adoptan los modelos de doble flujo, asimétricos o simétricos, funcionan como consultores o gestores estratégicos de comunicación.

Los roles, pues, se convierten en indicadores del poder de los departamentos de las organizaciones y, según el nivel de poder concedido al profesional, es posible determinar el ámbito probable de su actuación en el proceso decisorio al lado de los altos directivos.

La tipología desarrollada por Broom e Dozier (1986) sugiere cuatro roles: técnico de la comunicación, especialista en las relaciones con los medios de comunicación, consejero y administrador de la comunicación. En el primer rol -técnico de la comunicación- el profesional ejecuta tareas tácticas de comunicación y no participa de los procesos decisorios de la empresa. El especialista en los medios de comunicación, en cambio, se dedica a mantener relacionamientos estables y duraderos con los medios con el objetivo de promover la organización.

Tampoco en ese caso existe la participación del profesional en las decisiones estratégicas. El consejero ejerce un poder e influencia relativos en los procesos decisorios siempre y cuando el tema en que esté involucrado sea pertinente al área de la comunicación.

El cuarto rol corresponde al administrador de la comunicación; en éste, el profesional elabora e 
implementa las políticas de comunicación de la organización; además, integra la alta administración en el proceso de toma de decisiones estratégicas de la empresa.

Wright (1995) admite un quinto rol para los profesionales de relaciones públicas, el de ejecutivo de la comunicación, quien actúa como parte del staff corporativo, en funciones de vicepresidente o director, directamente responsable ante el CEO de la compañía.

\section{Cultura nacional y cultura organizacional}

La cultura es parte fundamental de todo grupo social. Así, para que sea posible comprender su función clave en las organizaciones, hay que identificar la cultura nacional para luego definir la cultura organizacional.

La cultura nacional es el conjunto de características de una población, dentro del límite de un país, que distingue a una sociedad de las demás; en ella los individuos están congregados en torno de sistemas comunes tales como jurídico, educacional, económico, político y lingüístico. Resulta esencial, pues, es estudiar la cultura nacional para entender por qué las personas en las organizaciones se comportan de manera distinta en diferentes países.

La cultura organizacional, en cambio, es un conjunto de manifestaciones tangibles e intangibles compartidas por los miembros de una organización. Es producto de la historia y determina las creencias, valores y comportamientos que a menudo son adoptados inconscientemente. Puede ser considerada como el patrimonio social de experiencias vividas y acumuladas por una organización y transmitidas a las nuevas generaciones a través de los procesos de aprendizaje. Según Freitas (1991, p. 34), "las organizaciones pueden ser vistas como fenómenos de comunicación, cuyo ejercicio ayuda a crear una cultura organizacional”. Así, comunicación y cultura se influyen mutuamente. $\mathrm{Si}$, por un lado, la comunicación es un elemento fundamental de la cultura -sin la cual no existiría- por otro lado, los medios de comunicación están fuertemente condicionados a un determinado contexto cultural.

Hemos así tenido que identificar los elementos que constituyen la cultura nacional y organizacional como el primer paso en nuestro intento de entender el proceso de implementación de los modelos de gestión y de comunicación en las organizaciones brasileñas y chilenas en nuestro estudio.

\section{Características de la cultura organizacional brasileña}

La sociedad brasileña sufrió transformaciones profundas en las últimas dos décadas del siglo XX. En ese período Brasil completó la transición del régimen dictatorial para el orden democrático, y de un nacionalismo proteccionista para un nuevo orden económico globalizado. Los medios de comunicación se transformaron en espacio de diálogo tras décadas de censura, y las organizaciones empezaron a sentir la presión del entorno externo para modernizar sus modelos de gestión para sobrevivir.

En el estudio realizado, los rasgos culturales identificados en las organizaciones brasileñas según la descripción de Barros y Prates (1996), corresponden a un "estilo brasileño de administrar", y que se pueden dividir en cuatro subsistemas: 


\section{Subsistema de los líderes}

a) Concentración del poder: basado en la autoridad y tradición, lo que resulta en distancia jerárquica y estructuras de poder autocráticas;

b) Personalismo: que enfatiza el carisma personal y valora la mantención de relacionamientos;

c) Paternalismo: combinación de concentración de poder y de personalismo; con una fuerte herencia de los terratenientes, con la extensión del dominio del espacio privado hacia el espacio público.

\section{Subsistema institucional}

a) Postura de espectador: baja conciencia crítica de los liderados, poca iniciativa y capacidad de realización, transferencia de responsabilidad para el otro;

b) Formalismo: las reglas sirven para regular las relaciones entre líderes y liderados, para lograr un mecanismo de estabilidad y equilibrio;

c) Impunidad: fortalece la posición de poder; la ley existe para el más débil y los derechos individuales son monopolio de pocos.

\section{Subsistema personal}

a) Lealtad a las personas: hacer parte del grupo para lograr aceptación o prestigio; la lealtad también está relacionada con la ética personal, humaniza las relaciones y busca la armonía.

b) Evita el conflicto: normalmente el conflicto es tratado en círculos cerrados, con soluciones indirectas, más comúnmente en el sentido líder - liderado.

4. Subsistema de los liderados, con un rasgo:

a) Flexibilidad: es la versión moderna del proceso llamado de 'manera criolla'; puede ser interpretada como la espontaneidad y la intuición; ayuda a las personas a adaptarse en situaciones de incertidumbre o de crisis.

\section{Características de la cultura organizacional chilena}

En la segunda mitad de la década de 1980, Chile inició un importante proyecto de reforma económica que produjo repercusiones en las áreas política y social; el auge del proceso democrático fueron las elecciones libres en 1990. Tras 17 años de una dictadura que dejó profundas huellas en los ciudadanos, la sociedad empezaba a vivir un período de transición política, económica, cultural y social. Chile ya no era el mismo país del año 1973. Los cambios que se fueron produciendo a lo largo de la última década del siglo XX trajeron nuevos hábitos, costumbres y valores que fueron prácticamente impuestos por el fenómeno de la globalización y por la presión externa de países vecinos y de otros continentes.

La cultura organizacional chilena se fue acomodando a ese nuevo escenario; hoy Chile es considerado uno de los países más prominentes en América Latina en términos políticos, económicos, sociales y culturales.

Para Rodríguez (1998), 
es importante señalar la importancia de la cultura de una sociedad en los aspectos de la organización, pues existen bases culturales en la formulación de un modelo de gestión de empresas en y para Chile. Es importante verificar como la cultura refuerza los comportamientos y valores según su forma particular de entender el mundo y los nuevos tiempos (Rodríguez, 1998, p. 36).

Durante la investigación utilizamos la tipología desarrollada por Rodríguez (1992, 2000) como resultado de experiencias acumuladas como consultor e investigador en el área. El autor menciona cuatro principales características de la cultura organizacional chilena, a saber:

a) Paternalismo: valor central para los latinoamericanos; forma muy personalizada de relación entre un superior que protege y un subordinado que le rinde lealtad;

b) Respeto: los trabajadores otorgan gran importancia al respeto: en el trato entre los trabajadores y en la relación con sus superiores; esperan, además, ser tratados con respeto;

c) Desconfianza: una de las características más relevantes del campesino es la desconfianza ante lo desconocido; es una actitud expectante y alerta ante cualquier proposición que no esté muy clara y directa;

d) Individualismo: el individualismo está presente en la a medida que asciende el nivel educacional y los modelos de gestión obligan al individuo a ser más competitivo, incluso porque Chile es un país pequeño y limitado en términos de mercado de trabajo calificado.

Curiosamente, las características de la cultura organizacional chilena apuntadas por Rodríguez tienen cierta similitud con las características de la cultura organizacional brasileña presentadas por Barros y Prates. Es importante observar que los rasgos culturales están en constante transformación y evolución, y que algunas características pueden ser más resistentes, mientras otras sucumben más rápidamente a la presión externa.

\section{Escala de valores organizacionales validada por Tamayo (1994) y complemen- tada por Rodríguez $(1991,1995)$}

Muchos investigadores se han dedicado a sugerir cómo debería ser la práctica de relaciones públicas (teoría normativa); sin embargo, pocos estudios se ocupan de determinar por qué diferentes organizaciones tienen prácticas distintas de relaciones públicas (teoría positiva). Para verificar el grado de importancia de la función de relaciones públicas en las organizaciones, es esencial conocer los valores organizacionales que las orientan, pues Mintzberg (1983) señala que es la alta dirección que identifica, establece y disemina los valores corporativos a través de sus políticas, objetivos y metas. 
Los valores, además de orientar la vida de las personas individualmente, también tienen la función de guiar la vida de la organización y el comportamiento de sus miembros.

Zippo (1983) revela que proyectos de investigación con CEO's de empresas norteamericanas demuestran que los valores son mayormente construidos por la alta dirección, y que están vinculados a los objetivos organizacionales. Mintzberg (1983) también revela que el CEO es el ejecutivo más poderoso de toda la jerarquía dentro y fuera de la organización; y es él que, además de tener el poder formal basado en la legitimidad de su rango y competencia, personifica la organización y modela su ideología.

Aidar y Alves (1997) añaden que en las organizaciones brasileñas existe una diferencia entre el repertorio de los directivos y aquel de los demás trabajadores, que resulta en la distancia de poder entre los niveles jerárquicos, un rasgo de la cultura brasileña.

Un análisis de la cultura chilena, sin embargo, permite observar que la estratificación social también se refleja en el mundo del trabajo, según señala Rodríguez (1991, 1995, 2000).

De la jerarquía de valores presentadas por los referidos autores, elegimos los siguientes para verificar la relación con los modelos de práctica y el rol desempeñado por los profesionales: maximización de lucros, eficacia, honestidad, creatividad, innovación, jerarquía, comprometimiento, respeto al trabajador, eficiencia y competencia profesional.

\section{¿Cómo funcionan las organizaciones chilenas y brasileñas?}

En términos del modelo de relaciones públicas practicado en los países investigados, hemos constatado el predominio del modelo asimétrico de doble flujo y, en menor escala, del modelo simétrico de doble flujo.

La visión asimétrica es la más diseminada precisamente porque el concepto de comunicación adoptado está equivocado; en esos casos, la comunicación es entendida como herramienta, y se utiliza la persuasión para que los públicos acojan las políticas organizacionales como una manifestación de poder y liderazgo. Las organizaciones brasileñas y chilenas siguen adoptando una postura reactiva hacia sus públicos, utilizando una estructura vertical -desde la cumbre del poder hacia los públicos- para enviar mensajes que buscan la aceptación de su misión y de sus propósitos. Esas organizaciones cultivan su 'imagen' basicamente a través de contactos con los medios, y muy pocas veces se preocupan en evaluar la percepción de los públicos hacia ellas. En situaciones de crisis las organizaciones buscan otras formas de relacionarse con sus públicos y lo hacen, por ejemplo, por medio de encuentros con la comunidad, reuniones con asociaciones de categorías profesionales, entrevistas en la televisión y la radio. La utilización de esas herramientas ocurre porque generalmente tratan de 'proteger' su imagen, más que preservar sus públicos de un posible efecto de la crisis.

Cabe señalar que, con el advento de la tecnología, los medios digitales se han convertido en una de las vías más corrientes y proactivas para la diseminación instantánea de información sobre la organización. Hay que aceptar esta nueva realidad reconociendo su fuerza y creciente dominación, pero los medios digitales no reemplazan los tradicionales, puesto que los objetivos son distintos en términos de contenidos y mensajes.

Se observa, asimismo, que las características de la cultura nacional influyen en la cultura organizacional a medida que la centralización y la toma de decisiones están en manos de pocos eje- 
cutivos de la empresa, con poca o ninguna participación del profesional de relaciones públicas. Como se puede ver en las Tablas 1 y 2, los modelos de práctica de relaciones públicas están relacionados con la cultura organizacional y, en ambos países, $36 \%$ de las organizaciones brasileñas y $31 \%$ de las chilenas de la muestra se encuentran en un período de transición como consecuencia de la apertura económica y política a partir de los años 1990 y del fenómeno de la globalización. Hay que recordar, sin embargo, que los cambios culturales se procesan a lo largo del tiempo, y que a las empresas les ha costado mucho adoptar nuevos modelos de gestión, de liderazgo y de estructuras más participativas y menos autoritarias.

En las Tablas 1 e 2 se muestran algunos resultados de la investigación, identificándose con números a cada una de las 35 organizaciones encuestadas para garantizar la confidencialidad de la información.

¿Cuál es la relación entre la cultura y la actividad de Relaciones Públicas en las organizaciones brasileñas?

\begin{tabular}{|c|c|c|c|}
\hline Modelos & Cultura Autoritaria & $\begin{array}{l}\text { Per'odo de } \\
\text { Transici-n }\end{array}$ & Cultura Participativa \\
\hline Simžtrico de doble flujo & 2021 & 111516 & 010305081215 \\
\hline A simŽtrico de doble flujo & 0409101719 & 0206131422 & 07 \\
\hline \multicolumn{4}{|l|}{ Informaci-n Poblica } \\
\hline \multicolumn{4}{|l|}{ A gencia de Prensa/Divulgaci-n } \\
\hline & $32 \%$ & $36 \%$ & $32 \%$ \\
\hline
\end{tabular}

Tabla 1.¿Cuál es la relación entre la cultura y la actividad de Relaciones Públicas en las organizaciones brasileñas? Fuente. Ferrari, M. A. (2000).

¿Cuál es la relación entre la cultura y la actividad de Relaciones Públicas en las organizaciones chilenas?

\begin{tabular}{|c|c|c|c|}
\hline Modelos & $\begin{array}{c}\text { Cultura } \\
\text { Autoritaria }\end{array}$ & $\begin{array}{l}\text { Per'odo de } \\
\text { Transici-n }\end{array}$ & Cultura Participativa \\
\hline SimŽtrico de doble flujo & 28 & 2934 & $2325355^{\circ}-$ \\
\hline A simžtrico de doble flujo & 24322731 & 2633 & \\
\hline Informaci-n Poblica & 30 & & \\
\hline \multicolumn{4}{|l|}{ A gencia de Prensa/Divulgaci-n } \\
\hline & $46 \%$ & $31 \%$ & $23 \%$ \\
\hline
\end{tabular}

Tabla 2. ¿Cuál es la relación entre la cultura y la actividad de Relaciones Públicas en las organizaciones chilenas? Fuente. Ferrari, M. A. (2000) 
Las tablas 1 y 2 muestran que hubo una evolución en la comunicación de las organizaciones a medida que se han abandonado los modelos de un flujo, en que la comunicación es una simple herramienta de difusión de informaciones sin feedback. El hecho de que un 36\% de las empresas brasileñas y un $31 \%$ de las chilenas se encuentren en un período de 'transición' significa un avance en términos de postura, que resulta de la presión del ambiente externo, de cambios estructurales y de mentalidad del empresario y de sus ejecutivos.

Resulta importante precisar, además, que mientras más se practique el modelo simétrico, mayor es la probabilidad de que la cultura organizacional sea participativa, con mayor apertura a la innovación, la creatividad, el trabajo en equipo y el empoderamiento. Así mismo, la cultura organizacional participativa aparece como respuesta a un entorno más vulnerable, en que las organizaciones están más expuestas a la presión de activistas y a las amenazas del mercado.

Con base en los rasgos de las culturas brasileña y chilena que fueron evaluados, se puede afirmar que la concentración del poder, la gestión autoritaria, la tradición, el paternalismo, el formalismo y el respeto constituyen elementos determinantes de la estructura de las organizaciones latinoamericanas y, por ende, del modelo de relaciones públicas por ellas practicado.

Respecto al papel desempeñado por los profesionales de relaciones públicas en las organizaciones chilenas y brasileñas, los resultados revelan una ambigüedad entre el quehacer de los relacionistas públicos y de los periodistas, con fuerte predominancia del segundo sobre el primero, puesto que existe más claridad en el 'hacer' periodístico por parte de las empresas y de la sociedad.

En Chile, nueve de los trece responsables por los departamentos de Relaciones Públicas/Comunicación eran periodistas, tres ejecutivos eran de otras áreas y sólo un profesional tenía formación en relaciones públicas. En Brasil, ocho eran periodistas, tres profesionales de relaciones públicas y once profesionales de otras áreas. Una parte significativa de los ejecutivos responsables por los departamentos, por lo tanto, no tiene la formación básica en comunicación, sino en áreas como Administración, Ingeniería y Derecho.

Respecto al papel desempeñado, sólo siete de los 22 ejecutivos en Brasil y seis de los 13 ejecutivos chilenos actuaban como administradores de comunicación, participando activamente en la toma de decisiones.

Una constatación importante fue la divergencia entre la visión del ejecutivo de comunicación y la del CEO respecto del rol desempeñado por los comunicadores. Tanto en Chile como en Brasil, casi la mitad de la muestra de los CEO's identificó al responsable de comunicación como alguien que ejerce una función 'mediática y técnica; en cambio, los ejecutivos de comunicación confirmaron que su función es estratégica y se identificaron como consejeros y administradores de comunicación.

Este resultado revela una visión distorsionada del área de Comunicación que suelen tener los altos mandos. El pequeño grupo de CEO's que consideran los responsables de comunicación como administradores, pertenecían a organizaciones más vulnerables por sus servicios y productos, o por estar en un contexto muy conflictivo, lo que las obligaba a empoderar a sus ejecutivos de comunicación para actuar prontamente ante las situaciones de conflictos o crisis. Examinando el sector de las empresas de la muestra, se constató que algunas de éstas eran empresas recién privatizadas por el gobierno, o que pertenecían a sectores más estables, con posibilidades más reducidas de exposición a crisis u otras situaciones de riesgo. En ese contexto, la alta dirección tenía una visión más instrumental de la comunicación, y se observó que el 
espacio para que el comunicador pudiera ejercer su papel de administrador de la comunicación era poco reducido.

Otra interesante observación del proyecto de investigación corresponde a la correlación entre el modelo de gestión autoritaria o participativa, el grado de vulnerabilidad y el papel desempeñado por el profesional de relaciones públicas. El Gráfico 1 revela el posicionamiento de las organizaciones investigadas en términos de los tres elementos intrínsecamente relacionados.

\section{Gestión x Vulnerabilidad x Papel del Comunicador}

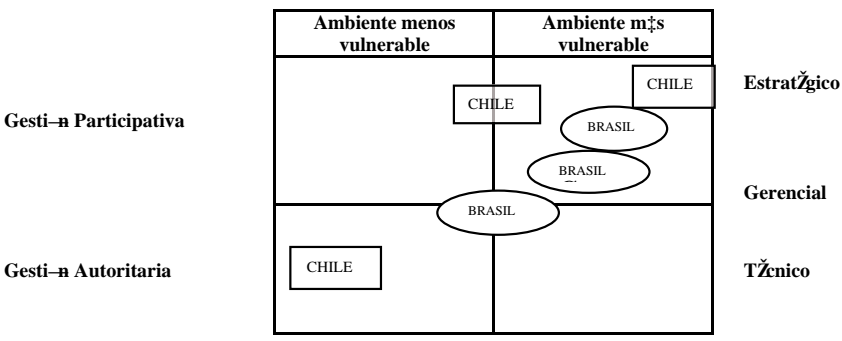

Figura 1. Gestión x Vulnerabilidad x Papel del Comunicador. Fuente. Ferrari, M. A. (2000).

Se observa que cuanto menor el grado de vulnerabilidad de la organización y de su entorno, menos espacio se abre desde la alta administración a los responsables de la comunicación (actúan como técnicos); por ende, se verifica que la gestión es más autoritaria y en ese caso desarrollando una comunicación más reactiva y mediática.

Por otro lado, las empresas que cuentan con productos y/o servicios más vulnerables y con un entorno más conflictivo empoderan a sus ejecutivos de comunicación para que éstos adopten prácticas más innovadoras y creativas para evitar crisis y situaciones de riesgo, y para responder a la presión de grupos de activistas y de consumidores. Pero, para que la función de administrador de la comunicación sea realmente practicada, las organizaciones deben tener en cuenta la comunicación como proceso y abrir espacio para que los profesionales participen en la toma de decisiones.

Ante un alto grado de incertidumbre o ambigüedad en el entorno externo, esas organizaciones más expuestas a los grupos de presión o de activistas elaboran programas de relaciones públicas más sofisticados; además, sus ejecutivos están empoderados por la alta administración para actuar rápidamente en la preservación de la reputación de la organización, con amplio conocimiento del negocio y con el buen relacionamiento con los públicos estratégicos.

La variable cultura fue aplicada en la encuesta para determinar su influencia en las prácticas de relaciones públicas y en los papeles desempeñados por los profesionales. Los resultados muestran que gran parte de las organizaciones se encuentra en transición de un modelo autoritario a uno más participativo, como resultado de las imposiciones del contexto externo y de la creciente actividad de los grupos sociales en la vida organizacional. 
Algunos rasgos de la cultura brasileña observados en los comportamientos de la alta administración como el paternalismo, el control de poder y el respeto a la jerarquía demuestran que la gestión se sigue ejerciendo con características autoritarias. En Chile, en cambio, la tradición, el respeto y el conservadurismo son las características que se identifican en las relaciones de trabajo. Las relaciones personales y sociales son altamente valoradas en Brasil, lo que refuerza el modelo de relación interpersonal; así, los lazos de amistad pueden, en algunos casos, garantizar el espacio profesional. La flexibilidad es otro rasgo distinguido en las organizaciones brasileñas, quizás como resultado de las muchas incertidumbres-económicas y políticas- que los brasileños han experimentado en las últimas décadas y que permite a los ejecutivos un grado más alto de adaptabilidad en diferentes escenarios. En algunos momentos, la flexibilidad se confunde con la 'cultura de la impunidad' o "corrupción" como resultado de la ausencia de ética y transparencia en los procedimientos organizacionales, mencionados por algunos de los encuestados como reflejos del comportamiento del Estado.

En Chile, al contrario, una estructura social de cariz más vertical que en Brasil, la distancia entre los trabajadores y sus superiores es más amplia, y existe menos espacio para los relacionamientos personales en el local de trabajo.

En cuanto a los valores compartidos en las organizaciones investigadas, es muy similar la clasificación de los valores en las organizaciones brasileñas y chilenas. La honestidad y el comprometimiento obtuvieron el más alto puntaje en ambos países. Se intenta fortalecer el valor honestidad, una vez que el comportamiento del Estado, principalmente en Brasil, está muy relacionado a la corrupción.

El compromiso se refiere al vínculo que las organizaciones tratan de mantener con sus trabajadores con el objetivo de aumentar la productividad. Esos dos valores son subyacentes a la comunicación de doble flujo y al análisis de escenarios que permite planificar a mediano plazo para evitar las crisis, mayormente en las organizaciones consideradas más vulnerables. En tercer lugar, la maximización de los lucros fue el valor más nombrado en las organizaciones brasileñas como necesario para su funcionamiento, nada más necesario en el momento de apertura de los mercados y de la competencia internacional.

Por otro lado, 'jerarquía' ha sido el valor con menor puntaje por las organizaciones en ambos dos países. Esto puede parecer un avance, puesto que cuanto menos se valora la jerarquía, se abre más espacio para que los organigramas sean más horizontales y, de esa manera, mayor será el espacio para el departamento de comunicación, y más fácil el relacionamiento junto a los públicos estratégicos; quizás el papel del responsable por el departamento camine hacia el nivel de administrador de comunicación.

El bajo valor atribuido a la jerarquía, además, está relacionado a la cultura nacional, a la libertad, la democracia y a los nuevos modelos de gestión que en ambos países pasaron a ser diseminados en las últimas décadas. En Chile, el valor eficiencia alcanzó en tercer lugar; podemos concluir que, juntamente con el compromiso los dos valores demuestran que las empresas están reforzando en sus trabajadores una postura y actitud positiva, comprometida con la misión y visión organizacional para mantener una performance superior frente a las demás empresas, resultado de la competencia global.

Creatividad e innovación fueron clasificadas como valores intermedios, lo que puede indicar que muchas organizaciones todavía no están preparadas para adoptar estrategias como empoderamiento, descentralización e innovación para conducir proyectos y planes. Para que esos 
valores sean incorporados en la práctica de las organizaciones será necesario una opción por una gestión más participativa, una cultura más abierta y la creación de un espacio para que la comunicación se transforme en un proceso proactivo, en que el profesional actúe como administrador de la comunicación, junto a la alta administración en la toma de decisiones.

Los datos presentados como resultado de la investigación llevada a cabo en el año 2000 siguen siendo validados en ambos los países, como puede ser observado por el estudio de opinión de la Facultad de Derecho de la Universidad del Desarrollo (Chile, 2011). Esta última investigación retrata a "Chile con un debilitamiento en la escala de valores y un claro descontento con el funcionamiento del estado de derecho"; situación también identificada en Brasil en los últimos años.

Como mencionamos al inicio de ese artículo, desde 2010 se está llevando a cabo una investigación sobre la práctica de las Relaciones Públicas en las empresas en los cinco continentes, auspiciada por IABC Research Foundation. Los primeros hallazgos referentes al estado del arte de las Relaciones Públicas en las organizaciones del continente latinoamericano demuestran que no ha habido un cambio sustancial en el comportamiento de las organizaciones hacia la comunicación.

Desde luego las organizaciones están más atentas en sus comunicaciones frente a los impactos que pueden causar en el entorno, pero en la mayoría de las veces lo hacen por mantener su imagen y reputación positiva y no por adoptar una visión simétrica que busca el equilibrio de objetivos tanto de sus públicos como de sus negocios.

Creemos que dado los cambios constantes y las presiones que las organizaciones han sufrido por parte del Estado y por medio de nuevas legislaciones y normas para la práctica de la sustentabilidad que la alta dirección pase a exigir que la comunicación sea tratada como función estratégica vital para su sobrevivencia en la sociedad.

\section{Reflexiones finales}

Es necesario reconocer que el eclecticismo es fundamental para conocer y entender la realidad de las organizaciones latinoamericanas, especialmente las chilenas y las brasileñas. Y porque la cultura está íntimamente asociada a la actividad organizacional, es imprescindible replantear los marcos de referencia externos. Poco sabemos sobre las organizaciones latinoamericanas, y aún menos sobre su cultura y modelos de comunicación.

La investigación expuesta en este artículo solo representa la 'punta del iceberg', y por eso resulta necesario continuar el estudio de las culturas nacionales para comprender la manera de administrar de las organizaciones, así como también identificar los procesos comunicativos y su relevancia para el quehacer organizacional. Sin embargo, resulta importante lanzar algunas conclusiones que pueden orientar el análisis del comunicador frente a su objeto de trabajo:

1. La visión del mundo de las sociedades latinoamericanas sigue siendo conservadora e influye en la manera de administrar los negocios;

2. Características como paternalismo, conservadurismo, centralización del poder, impunidad, desconfianza siguen muy presentes en las culturas nacionales de los países de la Región;

3. La baja participación de los profesionales de relaciones públicas en las organizaciones chile- 
nas y brasileñas es resultado de la definición poco clara de la actividad profesional; de esa manera, los ejecutivos o no conocen las relaciones públicas o no están convencidos de su importancia para sus negocios;

4. Las relaciones públicas están lejos de ser ampliamente practicadas en las organizaciones chilenas y brasileñas, principalmente en las medianas y pequeñas empresas;

5. Cuando las relaciones públicas cumplen una función más persuasiva y de convencimiento de los públicos de la imagen de la compañía, el modelo predominante es el asimétrico de dos manos;

6. El espacio de la comunicación en la organización está definido por la alta administración, de quien depende su grado de conocimiento del área;

7. Falta especialización a los profesionales de relaciones públicas para lograr ocupar puestos estratégicos en las organizaciones;

8. Los cambios económicos, políticos y sociales de los últimos años han permitido incrementar el espacio de las relaciones públicas; hay que aprovechar ese contexto para mostrar a las empresas el potencial de la disciplina;

9. Aunque de manera incipiente, las organizaciones han empezado a cambiar sus procesos de gestión y empiezan a preocuparse con asuntos como reputación, sustentabilidad y responsabilidad social, todos valores intangibles que influyen directamente en los negocios de la empresa y que se pueden conducir por relaciones públicas con mucha habilidad;

10. Muchas organizaciones miden los resultados del departamento de comunicación únicamente desde una perspectiva de costo-beneficio, cuando deberían analizar los logros intangibles que las relaciones públicas aportan al negocio.

\section{Notas}

1. Desde 2010 está en curso la investigación "Análisis de las Relaciones Públicas en los cinco continentes", auspiciada por IABC Research Foundation y desarrollada por Maria Aparecida Ferrari, Danny Moss, Fraser Likely, K. Sriramesh y Beert Reeger

\section{Referencias Bibliográficas}

Aidar, M. \& Alves, M. A. (1997). Comunicação de massa nas organizações brasileiras: explorando o uso da história em quadrinhos, literatura de cordel e outros recursos populares de linguagem nas empresas brasileiras. En, Motta, F. C. P. \& Caldas, Miguel P. (orgs.). Cultura organizacional e cultura brasileira. São Paulo: Atlas.

Barros, B. T. \& Prates, M. A. S. (1996). O estilo brasileiro de administrar. São Paulo: Atlas.

Broom, G. M. \& Dozier, D. (1986). Advancement for Public Relations role models. Public Relations Review, v.12, n.1, p.37-56.

Dozier, D. (1992). The Organizational Roles of Communications and Public Relations Practitioners. In Grunig, J. E. (org.) Excellence in Public Relations and Communication Management. Hillsdale, Lawrence Erlbaum.

Estudio de Opinión pública. (2011). Facultad de Derecho de la Universidad del Desarrollo. Dia- 
rio El Mercurio, Santiago, p. D 10.

Ferrari, M. A. (2010). La práctica de las Relaciones públicas en los cinco continentes. Proyecto de investigación auspiciado por IABC Research Foundation, Estados Unidos.

(2004). A trajetória das Relações Públicas nos países do Mercosul: reflexão e pesquisa da atividade. Paper presentado no NP 5 - Relações Públicas e Comunicação Organizacional. XXVII Intercom. (2000). A influência dos valores organizacionais na determinação da prática e do papel dos profissionais de relações públicas: estudo comparativo entre organizações do Brasil e do Chile. São Paulo, 2000. Tese (Doutorado em Ciências da Comunicação) - ECA-USP.

Freitas, M. E. de. (1991). Cultura organizacional: formação, tipologias e impactos. São Paulo: Makron, McGraw-Hill.

Freitas, A. B. de (1997). Traços brasileiros para uma análise organizacional. In: Motta, F. C. Prestes; Caldas, M. P. (orgs.). Cultura organizacional e cultura brasileira. São Paulo: Atlas.

Grunig, J. E.\& Ferrari, M. A. \& França, F. (2009). Relações Públicas: teoria, contexto e relacionamentos. São Caetano do Sul: Difusão.

Grunig, J.E.\& Hunt, T. T.(1984). Managing public relations. New York: Harcourt Brace Jovanovich College Publishers.

Mintzberg, H. (1983). Power in and around organizations. New Jersey: Prentice-Hall.

Rodríguez, D. M. (2001). Gestión organizacional: Elementos para su estudio. Santiago: Ediciones Pontificia Universidad Católica de Chile.

(1998). Aspectos culturales de la gestión de empresas chilenas. Santiago: Instituto de Sociología, Pontificia Universidad Católica de Chile.

Solorzano, E. \& Senac, R. (1999). Relaciones Públicas Superiores, Una Nueva Pedagogía. Lima: Universidad San Martín de Porres.

Tamayo, A. Hierarquia de valores transculturais e brasileiros (1994). Psicologia: Teoria e Pesquisa, Brasília, v. 10, n. 2, p. 269-285.

Van Ruller, B. \& Vercic, D. (2003). Perspectivas européias das relações públicas. Revista Comunicação \& Sociedade, Programa de Pós-Graduação em Comunicação Social: Universidade Metodista de São Paulo, 24, no. 39, P. 155 - 172.

Wright, D. K. (1995). The role of corporate public relations executives in the future of employee communications. Public Relations Review, 21 (3), 181-198.

Zippo, Mary. (1983). CEOs setting compensation and other policies to reinforce corporate culture. Personnel, p. 42-44, Nov./Dec.

Summary: The article examines the roles and the practices of Public Relations professionals in Brazilian and Chilean organizations. Through an analysis of the principal concepts of Public Relations and Communications, it is shown that the legitimacy of these areas is linked to local political, economic, social and cultural factors.

The results of the analysis named Influence of organizational values in practice and the role of public relations professionals: Comparative Study between organizations in Brazil and Chile by Ferrari (2000) reveal that both Chilean and Brazilian organizations are making a transition from authoritarian to participative cultures, which is associated to the phenomena of market globalization, new management styles, the opening of the economy, political stability and greater participation of the population in the life of an organization. Despite the evolution registe- 
red by organizations in Chile and Brazil in the last decade, the research results continue to be relevant, as confirmed by a new investigation project by Ferrari (2010) in course.

Key words: communication - culture - national culture - organization - organizational culture - organizational values - Public Relations.

Resumo: O presente texto examina a prática das relações públicas e do papel que desempenham os profissionais que administram a área em organizações chilenas e brasileiras. Aborda conceitos de comunicação e relações públicas para compreender que a legitimidade das áreas está associada a fatores políticos, econômicos, sociais e culturais locais. De acordo com os resultados da pesquisa, A influência dos valores organizacionais na determinação da prática e do papel dos profissionais de relações públicas: estudo comparativo entre organizações do Brasil e do Chile (Ferrari, 2000) boa parte das organizações, tanto no Chile como no Brasil, encontra-se na transição de uma cultura autoritária para participativa, uma evolução que está associada com o fenômeno da globalização dos mercados, dos novos modelos de gestão, da abertura econômica, da estabilidade política e de uma maior participação dos cidadãos na vida empresarial. Apesar do desenvolvimento alcançado na última década pelas as organizações no Chile e no Brasil, os resultados apresentados seguem válidos, como demonstra um estudo em andamento realizado por Ferrari (2010).

Palavras-chave: Relações Públicas - comunicação - cultura - cultura nacional - cultura organizacional - valores organizacionais - organização. 